\title{
Modelling maritime interaction in the Aegean Bronze Age
}

\author{
Carl Knappett ${ }^{1}$, Tim Evans $^{2} \&$ Ray Rivers ${ }^{2}$
}

The authors raise spatial analysis to a new level of sophistication - and insight - in proposing a mathematical model of 'imperfect optimisation' to describe maritime networks. This model encodes, metaphorically, the notion of gravitational attraction between objects in space. The space studied here is the southern Aegean in the Middle Bronze Age, and the objects are the 34 main sites we know about. The 'gravitation' in this case is a balance of social forces, expressed by networks with settlements of particular sizes and links of particular strengths. The model can be tweaked by giving different relative importance to the cultivation of local resources or to trade, and to show what happens when a member of the network suddenly disappears.

Keywords: Greece, Aegean, Bronze Age, maritime networks, spatial analysis

\section{Introduction}

If we follow Smith's line that 'spatial relationships are the sinews of archaeological research' (2003: 77), then we might expect spatial relationships to be fundamental across all scales of archaeological analysis. However, space has received a surprisingly uneven treatment within the discipline, and an assumed equivalence between physical, geometric space on the one hand, and relational, social space on the other, seems deeply entrenched. What is required is an approach that incorporates the fundamental notion that humans create space through social practices (Harvey 1973; 1996; Tilley 1994; Thrift 1996; Hetherington 1997; Smith 2003), while also acknowledging that physical parameters are not entirely redundant in this process.

In much spatial analysis physical interactions between points are seen as primary and the social interconnections come later (Batty 2005: 149). Sites are thought to emerge and gain their character on largely local grounds, and any interactions with other communities in the region follow on from that. The connections between sites are simply drawn as lines, without weight or direction. But the likelihood is that site interactions themselves contribute to the size and status of the sites in question (Sindbæk 2007). How, then, might we give the interactions and locations equal status and achieve, borrowing from Batty, an 'archaeology of relations'?

The behaviour of a large collection of entities, like island communities, is best addressed stochastically, rather than deterministically. Our approach synthesises techniques derived from statistical physics and complex network analysis for tackling social questions

1 Department of Art, University of Toronto, Canada (Email: carl.knappett@utoronto.ca)

2 Department of Physics, Imperial College London,UK (Email:T.Evans@imperial.ac.uk;R.Rivers@imperial.ac.uk)

Received: 11 June 2007; Revised: 27 February 2008; Accepted: 19 May 2008

ANTIQUITY 82 (2008): 1009-1024 
(Carrington et al. 2005; de Nooy et al. 2005; Evans 2005). However, before presenting our model we shall discuss its context, the southern Aegean Bronze Age.

\section{The study of networks in the Aegean}

Our case study for the development of the method starts with Broodbank's work on the Cyclades, the most systematic attempt thus far in Aegean prehistory to explain the growth of certain sites in terms of their interactions (Broodbank 2000). His approach was perhaps encouraged by the tiny resource base of some key Early Cycladic sites, making it likely that their role in a network was all important. In Broodbank's network, each individual site is represented by a node, and each connection between sites represented by a link. This simple transformation of the Cyclades into a graph of nodes and links enables a second step, the adoption of basic techniques from graph theory to analyse network characteristics. Broodbank opts for 'Proximal Point Analysis' (PPA), a technique already used effectively in archaeology and anthropology for interaction studies in other archipelagos, notably in Oceania (Terrell 1977; Irwin 1983; Hage \& Harary 1991; 1996).

Broodbank's next step is to add hypothetical sites to islands on the basis of population estimates derived from site surveys. Links are then drawn from each hypothetical site to its three nearest neighbours. Some sites emerge as more connected than others, with five or six links to other sites. These sites possess greater 'centrality' in the network, meaning that they might be expected to have a more prominent role in regional interactions. Comparing the results of his PPA with the archaeological data, of the five major Early Cycladic sites, three are 'central' in the PPA. Of course, Broodbank also has to suggest some motivation for these interactions - communities do not just interact without motives or goals. The Early Bronze Age Cyclades are agriculturally marginal and not self-sufficient and he cites basic demographic processes and the need for social storage networks (Broodbank 2000: 81-96), with power and prestige emerging consequentially out of network interactions.

\section{Enhancing the power of network analysis}

Broodbank's analysis is a rare example of an approach that succeeds in combining spatial and social factors. However, the Early Bronze Age Cycladic networks constructed by Broodbank are in many ways quite simple. First, the sites are assumed to be roughly equivalent in size, and the approximate homogeneity of the Cyclades means that the sites are distributed relatively evenly in space with little clustering. Secondly, the links between sites are also considered more or less equivalent: one node can connect with any other through a series of relatively short hops, implied by the available nautical technology (rowing).

However, during the later periods of the Middle Bronze Age of interest to us (c. 2000$1600 \mathrm{BC}$ ) the scale of interaction appears to change, with numerous connections apparent in the archaeological evidence between the Cyclades, Crete, the Greek mainland, the Dodecanese and coastal Asia Minor. This greater area includes large islands, such as Crete and Rhodes, and areas of mainland. Some long-distance links are unavoidable if a network covering this entire area is to remain fully connected. Furthermore, there appear to be 
substantial changes in transport technology between the Early Bronze Age and the later Middle Bronze Age, with the advent of the sail in $c .2000 \mathrm{BC}$. This throws into doubt one of the fundamental assumptions of PPA: that a site will necessarily link to its nearest neighbours. For while this might make excellent sense for rowing boats, the distances travelled under sail could easily increase by orders of magnitude. Lastly, archaeological evidence tells us that, in contrast to the Early Bronze Age, site size in the Middle Bronze Age varies widely, with substantial palatial towns on Crete and much smaller sites in the Cyclades and on the Greek mainland. In summary, any network model should be able to mimic this variability in link lengths, link direction and site size.

What do we want to know about these networks? There are two interesting features long recognised in the archaeological record but which have not been convincingly explained. The first concerns the size and centrality of Knossos, a palatial centre in north-central Crete. This site seems always to be central, and in the Middle and early Late Bronze Age is larger and apparently better connected than any other settlement in the south Aegean. Why? This question is normally answered in terms of its local resources and surplus, but we here consider it in terms of the wider properties of the interaction networks in which it participates. The second phenomenon of interest is 'Minoanisation' (see Broodbank 2004 for a recent review; see also Knappett \& Nikolakopoulou 2005; 2008). In this process a number of sites across the south Aegean, on both islands and mainland, develop increasingly complex exchange links and shared cultural traits. The driving force behind this is the large island of Crete, with certain central sites, and Knossos in particular, seemingly most involved. While some studies have sought to explain this phenomenon in interactionist terms (e.g. Davis 1979; Berg 1999), they have not done so explicitly using network models composed of nodes and links.

These two phenomena, the centrality and size of Knossos and the growth of Minoanisation, may be related. Minoanisation sees the emergence of relatively long-distance links (Knossos to Thera, for example, is $100 \mathrm{~km}$ ). Such links are presumably 'expensive' in some way; and we might assume that a large site with more resources has a greater chance of maintaining such a link than does a small site. And this forces us to realise that if a network is indeed to be sustained over a large asymmetrical grid of this kind, then large sites are likely to feature prominently. We may further assume that if these networks have a purpose for acquiring resources, and information about resources - then large sites are much more likely to target other large sites in that quest. This means that there are also 'gravitational pulls' to be taken into account when we examine such networks - the tendency of like to seek out like. This is the proposition that we now examine through network analysis.

Our modelling approach has to satisfy a few basic needs. First, it must incorporate some sense of function: regional interaction networks must accrue some benefit, balanced against their costs. Hence our model works on the assumption of some basic optimisation. Secondly, we want our model to take account of geographical distances while not being strictly determined by them. Thirdly, we want to be able to articulate scales in a sensible manner; our model is neither fully bottom up like agent-based modelling, which tends to aggregate scales very coarsely, nor entirely top down; it is set up in such a way that we can assess the interactions between the level of the site and that of the network as a whole. 


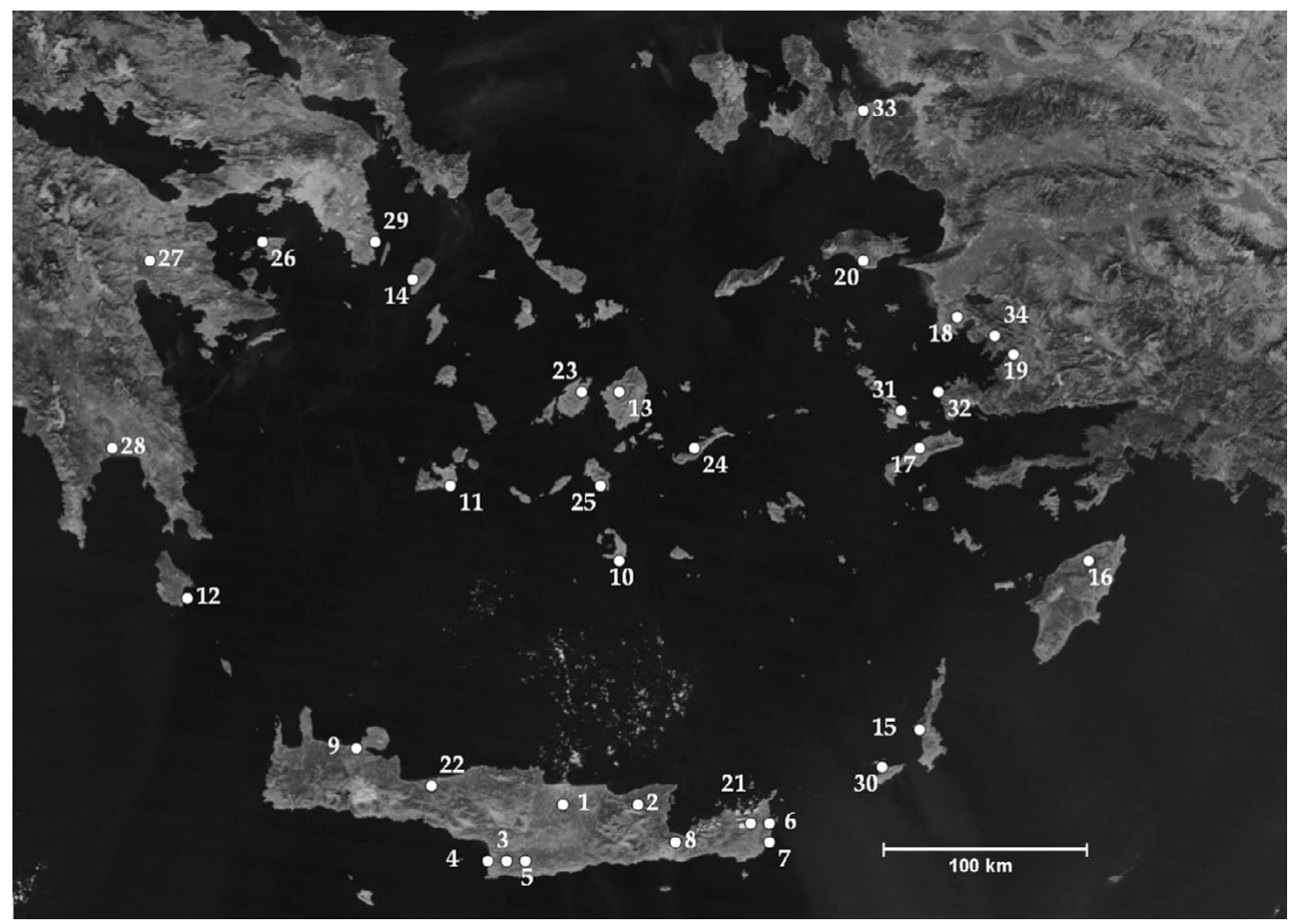

Figure 1. The location and numbering of the 34 sites used for our investigations, key given in Table 1 .

\section{The new model: imperfect optimisation}

The 34 sites shown in Figure 1 have been chosen on the basis of archaeological data. The evidence for each of them is not of equal standing; Crete, for example, has been more intensively researched than the other areas represented, i.e. the Cyclades, the Dodecanese, Asia Minor and mainland Greece. Nonetheless these 34 sites constitute a reasonable approximation of what would have been the most significant settlements in this period. One objection to our approach is that the archaeological data may be patchy; we may miss sites or not know their true size in this period. However, as we shall show, certain features of our model (gravity features) minimise the effects of our ignorance about individual sites.

\section{Variables}

We let a site be denoted by the numerical label $\boldsymbol{i}(\boldsymbol{i}=1,2, \ldots)$, and each site is assigned a fixed intrinsic carrying-capacity termed $S_{i}$, reflecting its local resources. We then assign to each site a variable $\boldsymbol{v}_{\boldsymbol{i}}$ that denotes that site's relative importance. Whereas $\boldsymbol{S}_{\boldsymbol{i}}$ is a given in the model, the value of $\boldsymbol{v}_{\boldsymbol{i}}$ for each site is part of the output. The total 'size' of a site (be this in terms of population, political importance or whatever) is then the product $\left(\boldsymbol{S}_{\boldsymbol{i}} \boldsymbol{v}_{\boldsymbol{i}}\right)$. So small rocky islands will have small $\boldsymbol{S}_{\boldsymbol{i}}$, yet they might maintain an occupation fraction 
Table 1. Key to sites used.

\begin{tabular}{|c|c|c|}
\hline 1. Knossos & 13. Naxos & 25. Ios \\
\hline 2. Malia & 14. Kea & Aegina \\
\hline 3. Phaistos & 15. Karpathos & Mycenae \\
\hline 4. Kommos & 16. Rhodes & Ayios Stephanos \\
\hline 5. Ayia Triadha & 17. Kos & Lavrion \\
\hline 6. Palaikastro & 18. Miletus & Kasos \\
\hline 7. Zakros & 19. Iasos & Kalymnos \\
\hline 8. Gournia & 20. Samos & Myndus \\
\hline 9. Chania & Petras & Cesme \\
\hline 10. Akrotiri & 22. Rethymnon & 34. Akbuk \\
\hline 11. Phylakopi & 23. Paroikia & \\
\hline 12. Kastri & 24. Amorgos & \\
\hline
\end{tabular}

bigger than one, $\boldsymbol{v}_{\boldsymbol{i}}>1$, if they play a pivotal role in the global network. We believe this is appropriate since the archaeological record for the Middle Bronze Age Aegean shows large variations in site size and importance. In the first instance, we have treated all sites as a priori equal, i.e. identical $\boldsymbol{S}_{i}$.

As for the links between sites, these too are given both physical and relational values in our model. We give as an input, $\boldsymbol{d}_{\boldsymbol{i}}$, a measure of the physical separation from site $\boldsymbol{i}$ to site $\boldsymbol{j}$. So far we have used the actual distance between sites, but we could modify $\boldsymbol{d}_{\boldsymbol{i}}$ to take account of difference between land and sea transportation, prevailing winds and currents and so forth. The relational strength of each connection is modelled using a second type of output variable, $\boldsymbol{e}_{\boldsymbol{i} j}$. This represents the fraction of effort that site $\boldsymbol{i}$ puts into its interaction with site $\boldsymbol{j}$ (and $\boldsymbol{e}_{j i}$ for the importance of the link going in the other direction, from site $\boldsymbol{j}$ to site $\boldsymbol{i}$ ). This is an improvement on most other archaeological network models, such as PPA, where the analysis makes no use of the properties which could be assigned to links.

\section{The social potential, or cost/benefit function}

Given our geographical inputs, how do we determine our population/relational outputs? One way to do this is to assume that these will, in some way, optimise the properties of the network overall. Explicitly, we assign a 'cost/benefit' social potential $\boldsymbol{H}$ to each possible configuration of site and link variables. We take it to have the form:

$$
H=-\kappa R-\lambda E+j P+\mu T
$$

$\boldsymbol{H}$ is a quantitative tool that allows us to assess the balance between the costs of supporting local population $(\boldsymbol{P})$, the benefits of exploiting local resources $(\boldsymbol{R})$ and the costs $(\boldsymbol{T})$ and benefits $(\boldsymbol{E})$ of maintaining links. $\boldsymbol{\kappa}, \boldsymbol{\lambda}, \boldsymbol{j}$ and $\boldsymbol{\mu}$ are constants, denoting the relative importance of each factor. By varying these constants we can derive and study different models. The details of $\boldsymbol{R}, \boldsymbol{E}, \boldsymbol{P}$ and $\boldsymbol{T}$ in terms of the variables given in the last paragraph (Figure 2) are given in the Technical Appendix. 


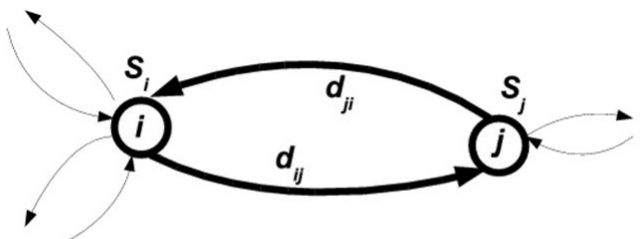

a. Inputs

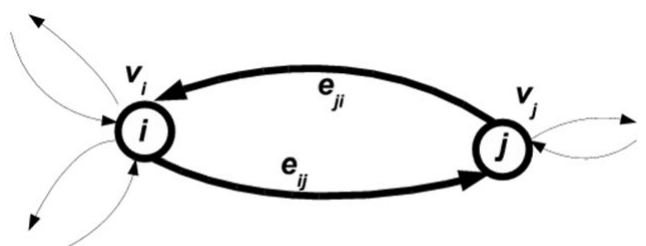

b. Outputs

Figure 2. The representation of our network. We show two sites, the circles labelled $\boldsymbol{i}$ and $\boldsymbol{j}$, together with the two links between these sites, the thick lines with arrows. a) Model inputs: each site has a fixed capacity designated $\boldsymbol{S}_{\boldsymbol{i}}$ and $\boldsymbol{S}_{\boldsymbol{j}}$, while the fixed physical distance from site $\boldsymbol{i}$ to site $\boldsymbol{j}$ is represented by $\boldsymbol{d}_{\boldsymbol{i}}$. Allowing for asymmetric winds, currents etc. means that the distance from site $\boldsymbol{j}$ to site $\boldsymbol{i}, \boldsymbol{d}_{\boldsymbol{j}}$, need not be the same. (b) Model outputs: the variable site occupation fractions are $\boldsymbol{v}_{\boldsymbol{i}}$ and $\boldsymbol{v}_{\boldsymbol{j}}$ while the relational aspect is captured by link variables $\boldsymbol{e}_{\boldsymbol{i}}$ for the fraction of effort site $\boldsymbol{i}$ puts into interactions with site $\boldsymbol{j}$. This is again asymmetric as site $\boldsymbol{j}$ may put a very different fraction of its trade into the link with site $\boldsymbol{i}$.

Through $\boldsymbol{H}$ we can both encode the obvious (e.g. there are penalties in over-exploiting resources) and the less obvious (e.g. contacts between kin groups). Most importantly, in constructing $\boldsymbol{E}$ we have adopted a gravity model (see Hodder 1974 and Renfrew 1975 for some archaeological applications of gravity models). Each pair of sites contributes a term proportional to the product of their populations multiplied by a decreasing function of the effective distance between them. This function is characterised by a daily distance $(\boldsymbol{D})$ which measures the scale of movement appropriate for sailing in the MBA. For distances $\boldsymbol{d}_{\boldsymbol{i} j}$ less than $\boldsymbol{D}$ this function is essentially 1 ; and for distances larger than $\boldsymbol{D}$ it effectively vanishes. We take $\boldsymbol{D}$ to be $100 \mathrm{~km}$ for sailing technology; by way of contrast we might imagine that $\boldsymbol{D}$ should be $10 \mathrm{~km}$ for a rowing-based Early Bronze Age simulation.

The lower the value of $\boldsymbol{H}$, the closer a network is to some optimal solution in which all the different constraints and interactions are balanced. We can picture $\boldsymbol{H}$ as providing an 'energy landscape'. Its spatial coordinates are our site and link variables, which specify a unique topology. The peaks and valleys of this landscape are then the 'energy' of the network. The aim is to try to calculate the values of $\boldsymbol{H}$ for different site and link values and find the global equilibrium, the set of values which reduce the overall energy of the network to its lowest level. The values of the output variables $\boldsymbol{v}_{\boldsymbol{i}}$ and $\boldsymbol{e}_{\boldsymbol{i}}$ for each site can then be read off. The constants $\boldsymbol{\kappa}, \boldsymbol{\lambda}, \boldsymbol{j}$ and $\boldsymbol{\mu}$ can then be reset with new values and the model constructed again. In effect this allows the researcher to set the emphasis - for example of the relative value of trade or domestic production and so derive and study new models.

A crucial ingredient in our approach is the way we look for our optimal network. We do not expect a real system to be in the perfect optimal state, and we recognise the valid criticisms of overly simple optimisation models (e.g. Johnson 1977: 480). We stress that, although our input is fixed, our model is not deterministic. For a given $\boldsymbol{H}$, there are typically numerous different solutions that approach the optimal solution. Hence our approach incorporates volatility, in the same way that a stock market might exhibit daily volatility; we expect short-term fluctuations in our networks, e.g. caused by annual weather variations. So, for one set of input parameters we find a different network every time we run our model. Our results must therefore be interpreted in a statistical sense. We see these variations as a positive feature, reflecting the imperfections and intrinsic short term volatility of real networks. 


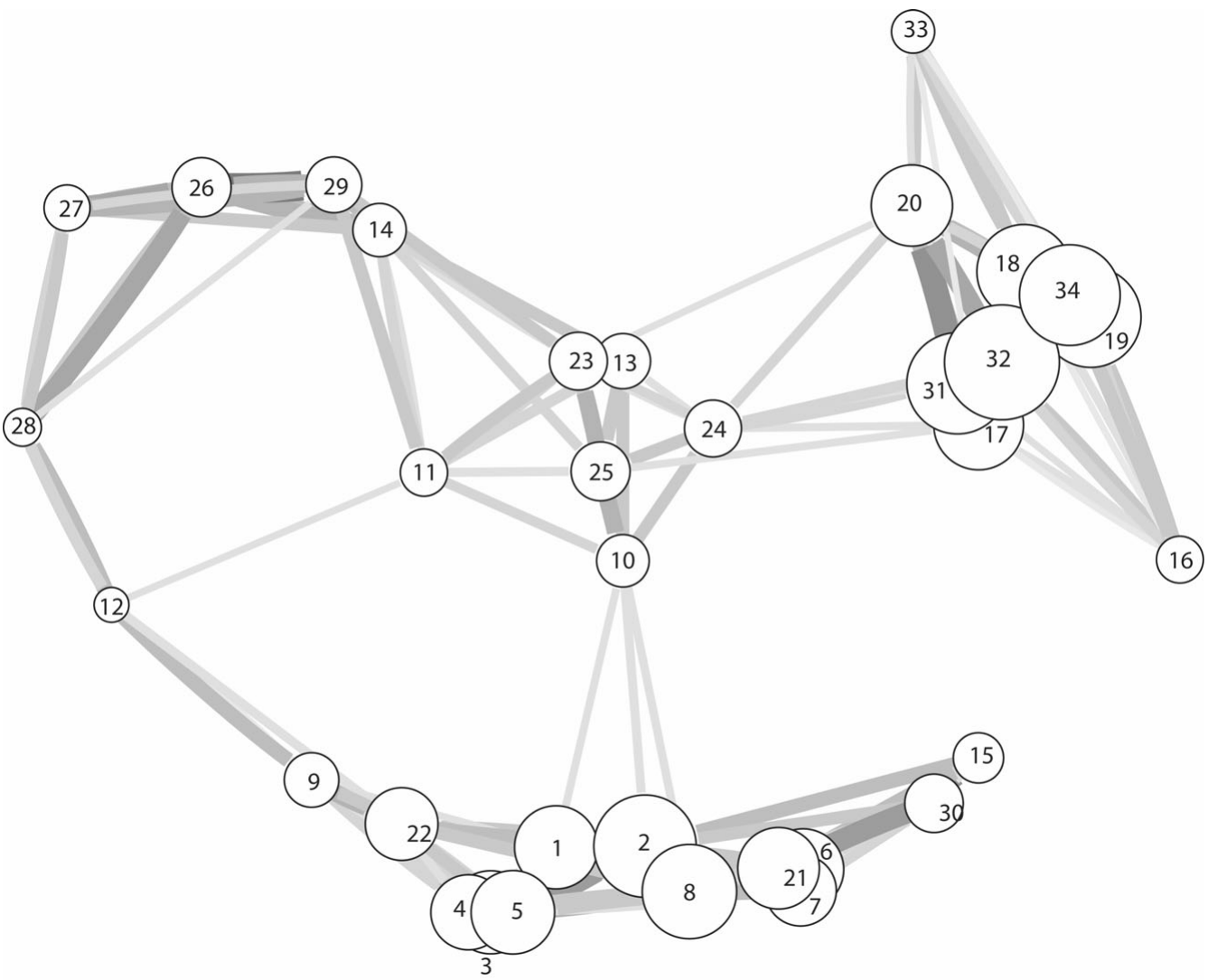

Figure 3. A network with each circle placed at the geographical location of a site, its radius proportional to the size $\left(\boldsymbol{S}_{\boldsymbol{i}} \boldsymbol{v}_{\boldsymbol{i}}\right)$ of that site. The darkness and thickness of the links represents the strength of interactions $\left(\boldsymbol{S}_{i} \boldsymbol{v}_{\boldsymbol{i}} \boldsymbol{e}_{i j}\right)$ but links which are $10 \%$ or less than the largest link are not shown $(\boldsymbol{\kappa}=1.0, \lambda=4.0, \boldsymbol{j}=0.5$, and $\boldsymbol{\mu}=0.1)$.

\section{Results}

The output of the model comes in two forms. The first, already mentioned, is the relative exploitation of resources $\boldsymbol{v}_{\boldsymbol{i}}$ or, equivalently, the absolute 'site size' $\left(\boldsymbol{S}_{\boldsymbol{i}} \boldsymbol{v}_{\boldsymbol{i}}\right)$ or population. The second is the absolute strength of inter-site links, $\left(\boldsymbol{S}_{\boldsymbol{i}} \boldsymbol{v}_{\boldsymbol{i}} \boldsymbol{e}_{\boldsymbol{i}}\right)$ for a link from site $\boldsymbol{i}$ to $\boldsymbol{j}$. In Figures 3-4 the sites are placed at their geographic locations (see Figure 1). Their relative sizes, as proposed by the modeling, are indicated by circles of different diameters and the importance of the links between them are represented by lines of different thickness and tone. In the first run of the model shown in Figure 3, which used particular input values for $\boldsymbol{\kappa}, \boldsymbol{\lambda}, \boldsymbol{j}$ and $\boldsymbol{\mu}$ as shown, we can see that the settlements in Crete, the Cyclades and the Dodecanese are all internally connected, but that there are only a few links between these main clusters. It is interesting from an archaeological perspective to see between which sites these links occur, and how strong they are. For example, it seems that in a number of different runs and in a relatively wide range of conditions, the link between the site of Akrotiri (site 10) on the island of Thera and a site on the north coast of Crete is important 


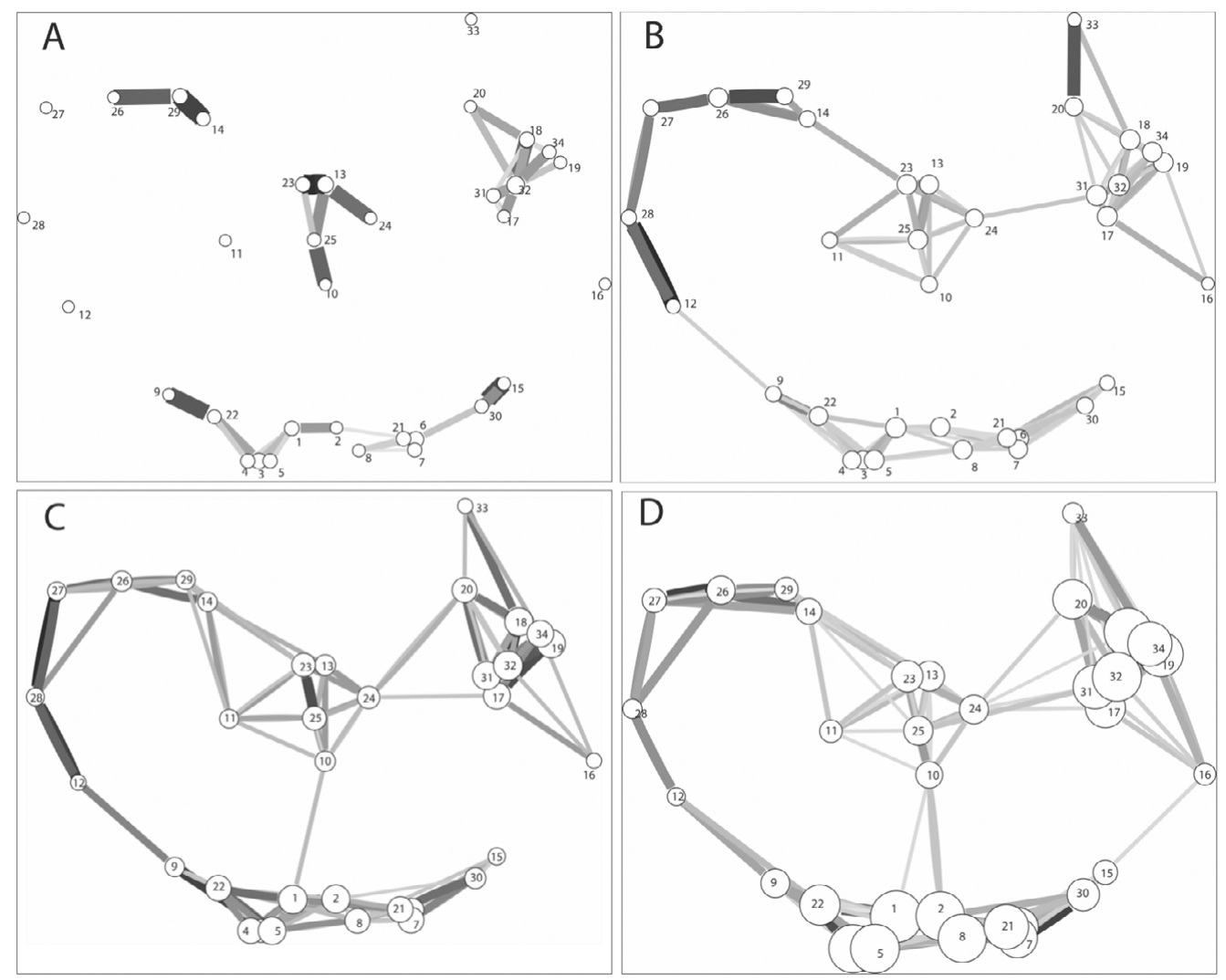

Figure 4. A sequence of four networks where the benefits of trade increase from $A$ to $D(\lambda=1.0,2.0,3.0$ and 4.0 but all with $\kappa=1.0, \boldsymbol{j}=0.5, \boldsymbol{\mu}=0.1)$. Note how the Akrotiri-central Crete link and other cross-cluster links increase. To emphasise the differences in site sizes the radius of each circle is proportional to the size $\left(\boldsymbol{S}_{\boldsymbol{i}} \boldsymbol{v}_{\boldsymbol{i}}\right)$ of the site it represents. The sites get bigger as interactions increase but so do the differences in sizes.

in keeping the network together. It does not seem to matter greatly whether that site is Knossos (1) or Malia (2).

This link is one of the first to disappear, however, when trade is 'penalised'. We can show this by gradually altering one of the input parameters and observing the outputs (Figure 4). Here we input an increasing value of $\lambda$, which effectively brings greater benefits for trade. We see how the Akrotiri-central Crete link becomes stronger, as do other cross-cluster links too, such as between east Crete and the Dodecanese. These are precisely the kind of links that seem to grow strong at the end of the Middle Bronze Age.

Another way we can represent these results is to show the same connections not geographically but topologically. Figure 5 shows the same network as in Figure 3 but its non-geographical layout emphasises some features in the network of interactions. For instance the pivotal role of the Cyclades and of Akrotiri in particular is reflected in the positioning of these sites.

A further way of representing the results that emerge is to study the correlations between various properties of sites. Figure 6 shows how the rank of a site varies with its size. Our 


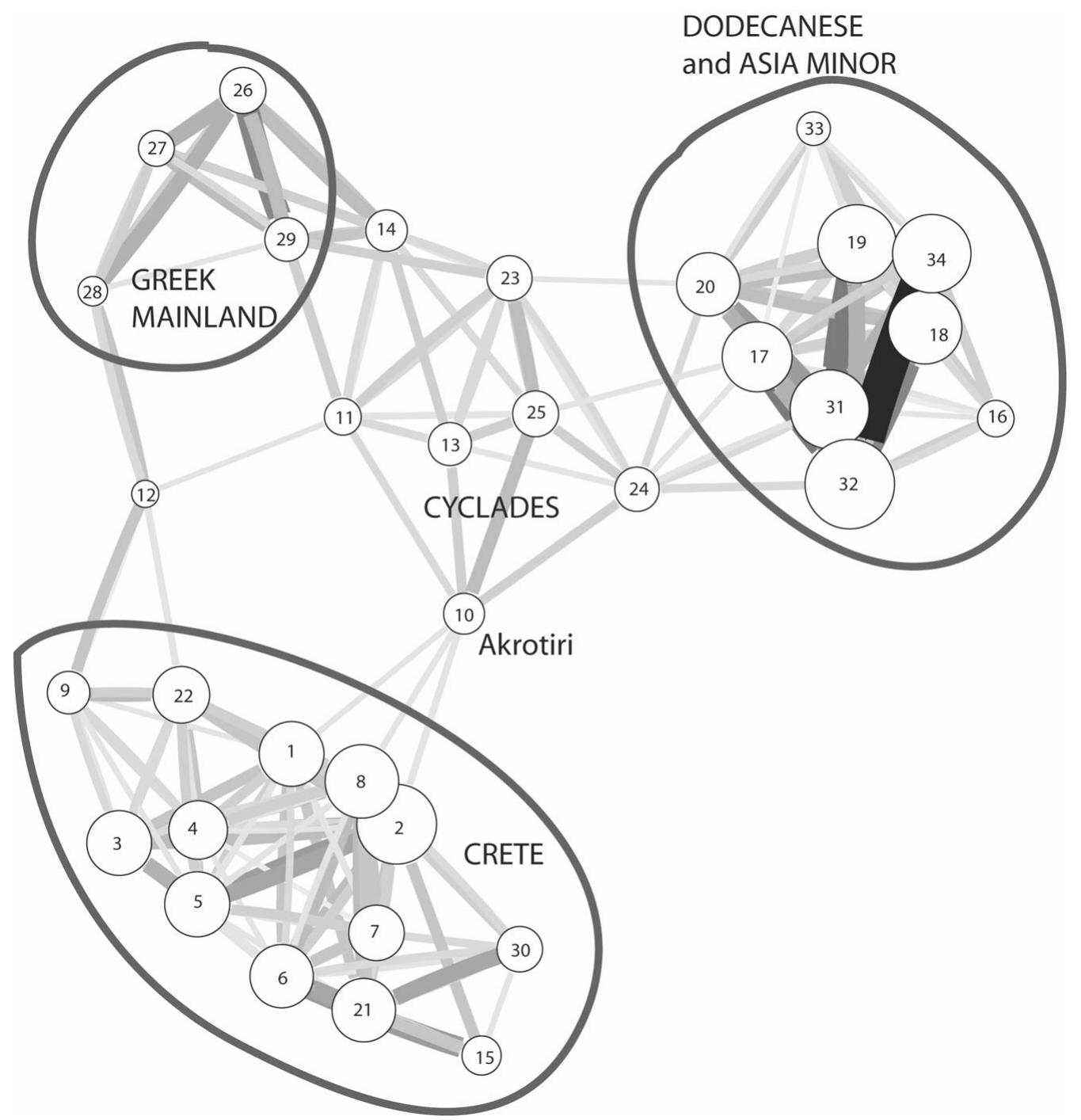

Figure 5. The same network as Figure 3 but shown laid out non-geographically (using the Kamada-Kawai method). Note how the central role of Akrotiri in the network is now reflected in its position in the figure.

definition of rank, a measure of a site's importance within the global network structure, is also the basis of Google's PageRank ${ }^{\mathrm{TM}}$. It is a measure of the 'traffic flow' through a site if journeys are made with probabilities proportional to the corresponding link variables $\boldsymbol{e}_{\boldsymbol{i} j}$. There is a general correlation between site size and rank, as one might expect in a gravity model. However, the gravity model here is sufficiently sophisticated to show that this is not a fixed relationship, and in some cases a site that is not that great in size may nonetheless rank highly in terms of network connectivity. For instance the large Dodecanese sites are usually found to be much less important to the network as compared to the Cretan sites of similar size. 


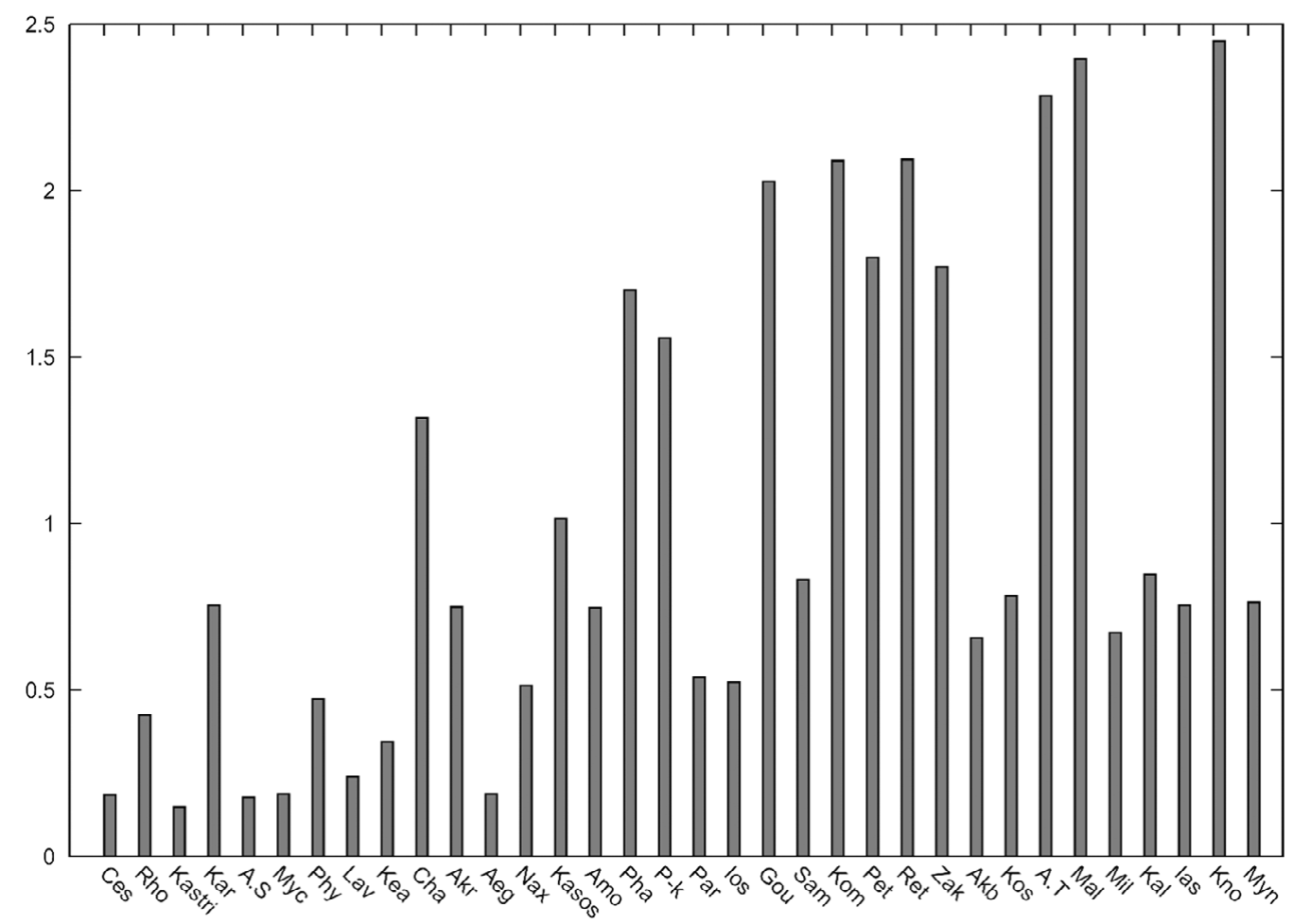

Figure 6. A histogram of site rank divided by site size, scaled relative to the average value over all sites. Rank is a measure of the global importance of the site in a network, found in much the same way as web sites are ranked by search engines. The sites are ordered from the smallest on the left to largest on the right. As there is a general trend for the values to incease as sites sizes increase, it shows that the largest sites have a slightly higher rank than their size would suggest. The most interesting feature though is the way that sites close to each other in the histogram, and so of similar size, can have greatly differing ranks. Measurements shown taken from the network of Figures 3 and 5.

\section{Discussion}

We opted here for a model in which large sites preferentially choose to interact among themselves. This follows the principle of homophily, pithily described in the phrase similarity breeds connection' (McPherson et al. 2001: 415). It seems that this kind of dynamic, also sometimes labelled 'assortative mixing', is common to many social networks (Newman 2002; Newman \& Park 2003). It thus seems reasonable to assume that this could have been a process at work in connecting communities across the southern Aegean.

This kind of dynamic is naturally implemented in gravity models such as ours. Further, gravity models also have the advantage of robustness: this is because the gravitational effect of several sites is (approximately) that of a single site at a distance. For example, we do include Knossos (site 1 in Figure 1 above) in our model, but not the nearby coastal site of Poros. In a gravity model we argue this is not a problem because we are working with an aggregate and not individual site details as such. This 'coarse graining' is thus a useful feature of our gravity model as it minimises the effects of our inevitable ignorance about individual sites, given the patchy nature of the archaeological record. 


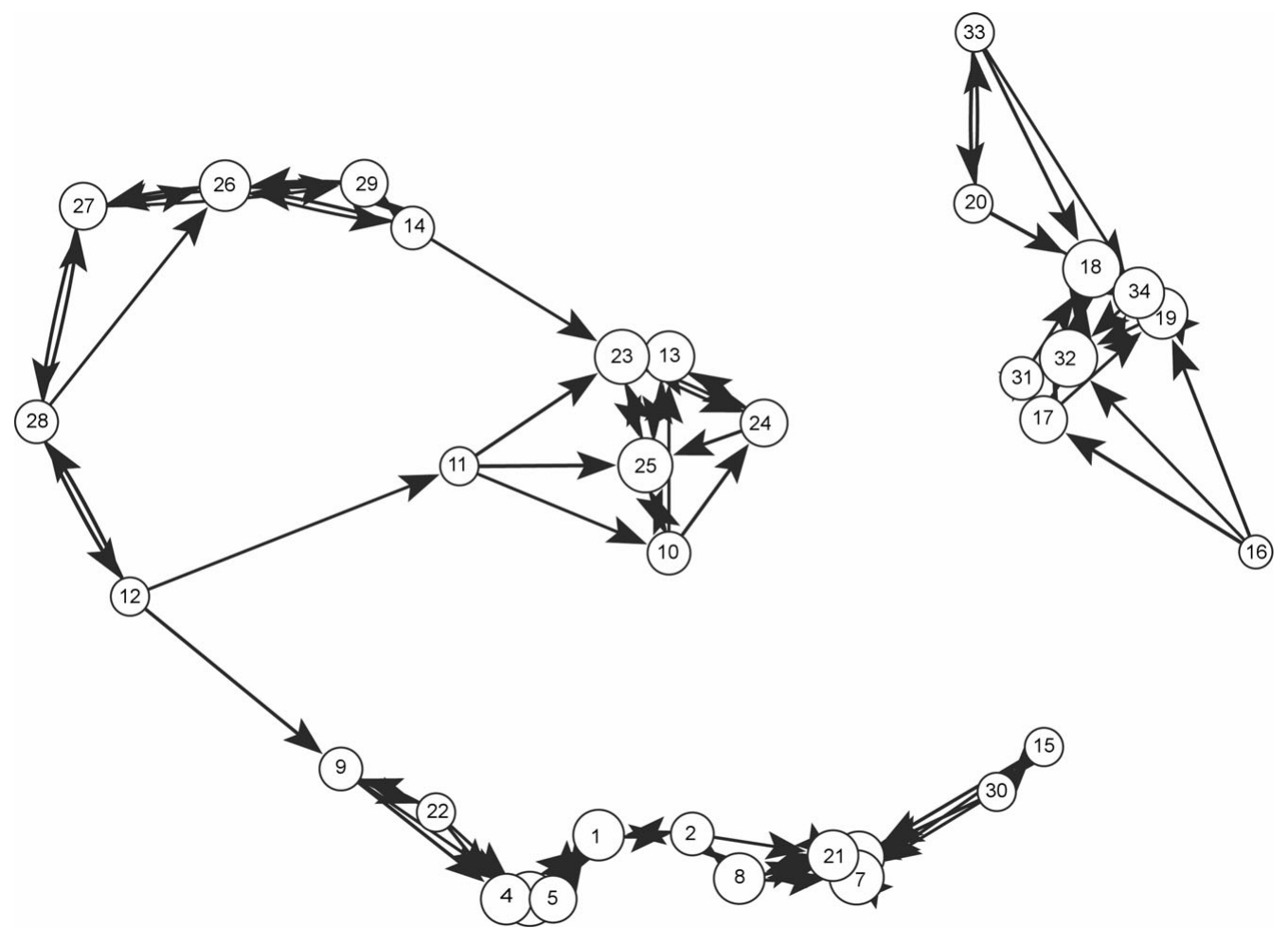

Figure 7. A PPA network where each site is given three links to the three sites closest to it. The size of the circles is proportional to the number of incoming links which varies from zero (e.g. Rhodes, site 16) or six for miletus (site 18) and Myndas (site 32).

We can return to PPA to underline the utility of the gravity model used here. A PPA of our 34 Middle Bronze Age sites is shown in Figure 7, with each site linked to its three nearest neighbours (as Broodbank did for Early Bronze Age). We can see that there are only indirect links between Crete and the Cyclades while the Dodecanese is completely isolated. This highlights the importance of the link between Akrotiri (10) and either Knossos (1) or Malia (2), which in each case is around $100 \mathrm{~km}$ long. These sites all have several closer neighbours within a $50 \mathrm{~km}$ radius so the Akrotiri-Knossos/Malia links are not shown up in PPA. Although such links are mildly penalised in our model, at the distance scale $\boldsymbol{D}=100 \mathrm{~km}$, they are not excluded. Indeed the appearance of Akrotiri-Knossos/Malia links implies there are wider network advantages to having such a direct link which overcomes any local disadvantages. In PPA only the number of sites or the links per site may be changed, so smooth long-term evolution is difficult to achieve and there is no short-term volatility.

A final, post hoc justification for our choice of a gravity model is that it has produced results commensurate with the record. In counterpoint, we have run two non-gravity 'asymmetric' models (although such models are sometimes called gravity models in the literature). In such models the strength of a link is determined only by the size of the site at one end of the link. In contemporary economic terms, a 'supply-side' model only considers the size of sites 
to which a link is made. This means that even a small site can gain a benefit by linking to a large site, but the link will not gain additional strength if the small site grows. This could be equivalent in Aegean terms to the growth of strong links between large Cretan sites and smaller Cycladic sites being driven solely from the Cretan side. However, in a 'demand-side' model, a site gains more benefit from links by having a large local population, regardless of the supply site sizes. In Aegean terms this would entail Cycladic sites growing larger in order to benefit from various links with other sites (irrespective of the size of those other sites). Unlike the gravity model (which reflects both supply and demand), these asymmetric models are sensitive to island site details. If, for example, we divide a large site in two, this would produce a different system. Thus asymmetric models are not robust, unlike our gravity model (with its aggregation of local site sizes). More importantly, asymmetric models do not seem able to produce the range in site sizes that is actually observed in the Middle Bronze Age Aegean.

The time evolution of the network is achieved by changing the parameters of the model to reflect changes in overall population and overall network activity. For instance, steady population growth might be studied by using the total value of our $\boldsymbol{S}_{\boldsymbol{i}} \boldsymbol{v}_{\boldsymbol{i}}$ parameters as a proxy for population. Most of the time the networks we find will evolve slowly along with the small changes we impose on our energy landscape. However, occasionally, a slight change in our energy landscape may provide a dramatic shift in the preferred networks produced, describing an instability or collapse. For example, a sudden physical change to the system, such as the destruction caused by the Theran eruption at the start of the Late Bronze Age, would be reflected by the elimination of Akrotiri. Remodelling the network before and after this event would show the consequences for its other members, for example a sudden decrease in the importance of Crete.

\section{Conclusions}

The gravity model seems to provide the results and robustness that one would hope for from a network model. It achieves stability in solutions between runs; it readily generates variation in site sizes and ranks; and it provides the kinds of long-distance links across clusters that we know existed in the Middle Bronze Age. Since we employ stochastic methods our model captures the intrinsic uncertainty of real systems, unlike the deterministic nature of PPA methods. This subtlety does require some sophistication in the model, but we have tried to keep it as simple as possible: our results depend only on $(\lambda / \kappa),(j / \kappa)$ and $(\mu / \kappa)$ and some physical input (daily transport distance $\boldsymbol{D}$ and physical distances $\boldsymbol{d}_{\boldsymbol{i}}$ ). We see this relative simplicity as an important advantage over Agent Based Modelling approaches (Bura et al. 1996).

We stress that we do not consider our model to be directly predictive. We are certainly not at a stage where we would believe all of the quantitative details generated by the model. Rather we see the evaluation of inputs and outputs as an exploratory process that allows us to articulate our questions in a qualitative fashion. The distinction made by Johnson (1977: 501) between prediction and understanding is apposite here: we have compared PPA, asymmetric and gravity models as a means of enhancing our understanding of inter-regional interaction, here for the Aegean Bronze Age. Specifically, the gravity model repeatedly shows that long-distance links between sites in north-central Crete (Knossos, Malia) and Thera 


\section{Carl Knappett, Tim Evans \& Ray Rivers}

(Akrotiri) are important in maintaining overall network connectivity. However, the model often produces only one link between these areas, and it does not seem to matter whether this is between Knossos and Thera or Malia and Thera. As one might expect in a gravity model, these north-central Cretan sites able to sustain long-distance links also tend to be among the largest sites. Site size and rank do tend to correlate strongly, but the model is also capable of producing 'anomalous' behaviour such that some pivotal sites of high rank are not particularly large. These anomalous sites, capable of 'punching above their weight', are often situated on the links between clusters; we may thus tentatively interpret them as 'gateway' communities (Hirth 1978).

Our results so far are interesting but further tests and improvements are needed. Our input distances $\boldsymbol{d}_{\boldsymbol{i}}$ should reflect actual transport times rather than physical distances. We could distinguish the capacity $\left(\boldsymbol{S}_{\boldsymbol{i}}\right)$ of existing sites and add new ones on the basis of cultivable area or probable population densities, following Broodbank's approach. Once we have our networks then we can test any conclusions we reach in a quantifiable manner, using approaches from statistical physics and analysing networks using the wide range of techniques available, such as 'random network walkers' to create site rankings or to assess the 'influence' of sites on others. Thus we are just at the beginning, but we hope nonetheless to have shown how the use of techniques from physics offers new means of assessing the various kinds of networks that have long preoccupied the social sciences. Far from using these tools for their own sake, we must ensure that our techniques are commensurate with the complexity of the archaeological data, and ensure any conclusions reflect changes in the details of our models.

\section{Acknowledgements}

We are grateful to the participants in various interdisciplinary EU-funded ISCOM (the Information Society as a Complex System) workshops over the past four years for their helpful comments on the ideas presented here. On the archaeological side, we warmly thank Cyprian Broodbank, Todd Whitelaw and Sander van der Leeuw for their insightful comments on earlier drafts, and Andy Bevan for his advice and help on GIS matters. The paper benefited considerably from the criticisms of reviewers Colin Renfrew and Mark Lake, and the invaluable advice of the Antiquity editor.

\section{References}

BATTY, M. 2005. Network geography: relations, interactions, scaling and spatial processes in GIS, in D. Unwin \& P. Fisher (ed.) Re-presenting GIS: 149-70. Chichester: John Wiley \& Sons.

BERG, I. 1999. The southern Aegean system. Journal of World-Systems Research 5(3): 475-84.

BroOdBAnK, C. 2000. An island archaeology of the early Cyclades. Cambridge: Cambridge University Press.

-2004. Minoanisation. Proceedings of the Cambridge Philological Society 50: 46-91.

Bura, S., F. Guerin-Pace, H. Mathian, D. Pumain \& L. SANDERS. 1996. Multiagent systems and the dynamics of a settlement system. Geographical Analysis 28(2): 161-78.
Carrington, P.J., J. SCOTT \& S. WASSERman (ed.). 2005. Models and methods in social network analysis. Cambridge: Cambridge University Press.

DaVIS, J.L. 1979. Minos and Dexithea: Crete and the Cyclades in the Later Bronze Age, in J.L. Davis \& J.F. Cherry (ed.) Papers in Cycladic prehistory (Monograph of the Institute of Archaeology 14): 143-57. Los Angeles (CA): Institute of Archaeology, University of California.

De Nooy, W., A. Mrvar \& V. Batagelj. 2005. Exploratory social network analysis with Pajek. Cambridge: Cambridge University Press.

Evans, T.S. 2005. Complex networks. Contemporary Physics 45: 455-74.

Hage, P. \& F. Harary. 1991. Exchange in Oceania: a graph theoretic analysis. Oxford: Clarendon Press. 
-1996. Island networks: communication, kinship and classification structures in Oceania. Cambridge: Cambridge University Press.

Harvey, D. 1973. Social justice and the city. London: Arnold.

-1996. Justice, nature and the geography of difference. Oxford: Blackwell.

Hetherington, K. 1997. In place of geometry: the materiality of place, in K. Hetherington \& R. Munro (ed.) Ideas of difference: social spaces and the labour of division: 183-99. Oxford: Blackwell.

HiRTH, K.G. 1978. Interregional trade and the formation of prehistoric gateway communities. American Antiquity 43(1): 35-45.

HODDER, I. 1974. Regression analysis of some trade and marketing patterns. World Archaeology 6(2): 172-89.

IRWIN, G. 1983. Chieftainship, kula and trade in Massim prehistory, in J.W. Leach \& E. Leach (ed.) The Kula: new perspectives on Massim exchange: 29-72. Cambridge: Cambridge University Press.

JoHnSON, G.A. 1977. Aspects of regional analysis in archaeology. Annual Review of Anthropology 6: 479-508.

Knappett, C. \& I. Nikolakopoulou. 2005. Exchange and affiliation networks in MBA southern Aegean: Crete, Akrotiri and Miletus, in R. Laffineur \& E. Greco (ed.) Emporia: Aegeans in East and West Mediterranean (Aegaeum 25): 175-84. Liège: University of Liège.
-2008. Colonialism without colonies? A Bronze Age case study from Akrotiri, Thera. Hesperia 77: 1-42.

McPherson, M., L. SMith-Lovin \& J.M. CoOK. 2001. Birds of a feather: homophily in social networks. Annual Review of Sociology 27: 415-44.

Newman, M.E.J. 2002. Assortative mixing in networks. Physical Review Letters 89(20): 208701. DOI: 10.1103/PhysRevLett.89.208701.

NeWMan, M.E.J. \& J. PARK. 2003. Why social networks are different from other types of networks. Physical Review E 68: 036122. DOI: 10.1103/PhysRevE.68.036122.

RENFREW, C. 1975. Trade as action at a distance: questions of interaction and communication, in J.A. Sabloff \& C.C. Lamberg-Karlovsky (ed.) Ancient civilization and trade: 3-59. Albuquerque (NM): University of New Mexico Press.

SINDBÆK, S.M. 2007. Networks and nodal points: the emergence of towns in early Viking Age Scandinavia. Antiquity 81: 119-32.

SMITH, A.T. 2003. The political landscape: constellations of authority in early complex polities. Berkeley (CA): University of California Press.

TerreLl, J. 1977. Human biogeography in the Solomon Islands. Chicago (IL): Field Museum of Natural History.

THRIFT, N. 1996. Spatial formations. London: Sage.

TiLley, C. 1994. A phenomenology of landscape: places, paths and monuments. Oxford: Berg.

\section{Technical Appendix}

Our 'gravity' model is defined by the following cost/benefit or 'energy' function (Hamiltonian):

$$
H=-\kappa \sum_{i} S_{i} v_{i}\left(1-v_{i}\right)-\lambda \sum_{i, j} V\left(d_{i j} / D\right) .\left(S_{i} v_{i}\right) \cdot e_{i j} .\left(S_{j} v_{j}\right)+j \sum_{i} S_{i} v_{i}+\mu \sum_{i, j} S_{i} v_{i} e_{i j}
$$

This can be abbreviated as:

$$
H=-\kappa R-\lambda E+j P+\mu T
$$

where $\boldsymbol{R}$ measures the exploitation of resources, $\boldsymbol{E}$ the benefits of maintaining links, $\boldsymbol{P}$ is the overall population and $\boldsymbol{T}$ the costs of maintaining links. The coefficients $\boldsymbol{\kappa}, \boldsymbol{\lambda}, \boldsymbol{j}$ and $\boldsymbol{\mu}$ measure the relative importance of these terms.

The sums are over the $\boldsymbol{N}$ different sites or over all possible pairs of sites, labelled by $\boldsymbol{i}$ or $\boldsymbol{j}$. Each site $\boldsymbol{i}$ has a fixed intrinsic carrying capacity $S_{i}$ and a variable exploitation fraction $\boldsymbol{v}_{\boldsymbol{i}}$ so that the total size of a site (representing its population, political importance etc.) is then $\left(\boldsymbol{S}_{\boldsymbol{i}} \boldsymbol{v}_{\boldsymbol{i}}\right)$. Carrying capacity $\boldsymbol{S}_{\boldsymbol{i}}$ is an input value we specify. Our variable $\boldsymbol{v}_{i}$ can be bigger than one but this will generally require a site drawing support from other sites via the network. In practice we limit $\boldsymbol{v}_{\boldsymbol{i}}$ to be less than $\boldsymbol{v}_{\max }$, a number we specify. If $\boldsymbol{v}_{\boldsymbol{i}}$ comes close to this cap, we rerun with a larger $\boldsymbol{v}_{\text {max }}$. 


\section{Carl Knappett, Tim Evans \& Ray Rivers}

The links from sites $\boldsymbol{i}$ to site $\boldsymbol{j}$ are associated with one constant input parameter $\boldsymbol{d}_{\boldsymbol{i} \boldsymbol{j}}$ and one variable $\boldsymbol{e}_{\boldsymbol{i} \boldsymbol{j}}$. The link variable $\boldsymbol{e}_{\boldsymbol{i}}$ take values between zero and one, representing the fraction of 'trade' or interaction ('influence') going from site $\boldsymbol{i}$ to site $\boldsymbol{j}$. This interpretation means that the total effect that site $\boldsymbol{i}$ has on site $\boldsymbol{j}$ can be thought of as $\left(\boldsymbol{S}_{\boldsymbol{i}} \boldsymbol{v}_{\boldsymbol{i}} \boldsymbol{e}_{\boldsymbol{i}}\right)$. It also means that we must limit the sum of link values emanating from each site $\boldsymbol{i}$ to be less than unity:

$$
\sum_{j} \boldsymbol{e}_{i j} \leqslant 1
$$

There is no demand that the link values $\boldsymbol{d}_{i j}$ or $\boldsymbol{e}_{\boldsymbol{i}}$ are symmetric so that these can represent various asymmetries in the physical and relational nature of links.

The first term proportional to a constant $\boldsymbol{\kappa}$ would control the size of sites if there were no outside contacts. It is the logistic map as used for simple models of population dynamics. The optimal fractional use of a site is $\boldsymbol{v}_{\boldsymbol{i}}=0.5$ which represents the idea that sites which are exploiting some but not all of their local capacity are likely to be optimal. However all relative sizes $0<\boldsymbol{v}_{\boldsymbol{i}}<1$, produce some benefit and it is only when $\boldsymbol{v}_{\boldsymbol{i}}>1$ do we deem that a site is too large for its local resources. Such values can only be achieved if supported by the interactions with other sites.

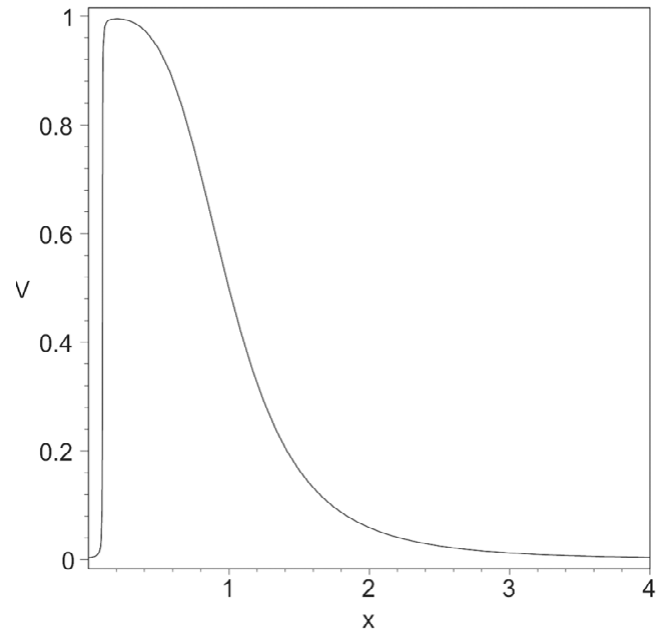

Figure 8. Interaction potential $V$ as a function of distance where $\boldsymbol{x}=\boldsymbol{d}_{i j} / \mathrm{D}$ with $\boldsymbol{D}_{v s}=\mathrm{D} / 10$.

hape of the potential function used for to important but so far we have worked only with the form $\boldsymbol{V}(\boldsymbol{x})=1 /(1+\boldsymbol{x})^{4}$ which gives the desired behaviour if $\boldsymbol{x}$ is the input physical distance $\boldsymbol{d}$ in units of $\boldsymbol{D}$, so $\boldsymbol{x}=\boldsymbol{d} / \boldsymbol{D}$. We also need to introduce a very short distance scale, $\boldsymbol{D}_{\boldsymbol{v}}$, which is the minimum separation required before we consider two sites to be separate entities. For sites separated by less than $\boldsymbol{D}_{\boldsymbol{v}}$ we set the potential to zero. All interactions between such close sites have zero value as any interactions are deemed to be counted in the internal structure of the location

Finally the last two terms are proportional to model parameters $\boldsymbol{j}$ and $\boldsymbol{\mu}$ multiplying factors which we may think of as the absolute total 'population' size and the absolute total 'trade' respectively. Increasing the parameters reduces the total value of the associated quantity, though the precise relationship is not simple. Thus we use these variables to control the population and trade and allow us to make comparisons between low and high population densities. Alternatively, we may think of $\boldsymbol{j}$ and $\boldsymbol{\mu}$ as the cost of adding one unit of population or trade to a network. interactions or 'trade' with one contribution for each direction between every pair of sites. It is proportional to the total 'populations' at both ends of a link $\left(\boldsymbol{S}_{\boldsymbol{i}} \boldsymbol{v}_{\boldsymbol{i}}\right)$ and to a link weight variable $\boldsymbol{e}_{\boldsymbol{i} \boldsymbol{j}}$. Because this term is proportional to the product of the population at each end of the link, this favours 'homophily' (McPherson et al. 2001). There is a final factor in this interaction term which is where we incorporate the effects of physical separation of the two sites. We do this through a 'potential' function $\boldsymbol{V}(\boldsymbol{x})$ (see Figure 8). This is essentially zero for long range long distance interactions give virtually no benefit and are unlikely to appear in our simulations; they are deemed to carry prohibitively high overheads. To define what we mean by a long distance we introduce another parameter $\boldsymbol{D}$. This is related to technological capabilities so we take $\boldsymbol{D}$ to be $100 \mathrm{~km}$ which is of the order of distance we expect to be able to travel using sail in the MBA. By way of contrast we might set $\boldsymbol{D}$ to be $10 \mathrm{~km}$ for a rowing-based EBA simulation. The

The second term, proportional to $\lambda$, allows for 
To summarise, if we had $N$ sites our model requires a total of $\boldsymbol{N}^{2}$ fixed physical input parameters representing the site 'size' through the capacities $\boldsymbol{S}_{\boldsymbol{i}}$ and site separation through the physical distances $\boldsymbol{d}_{\boldsymbol{i}}$. Values for these may be derived from data and we think of these parameters as given once we have defined our physical arena. The distance scales $\boldsymbol{D}$ and $\boldsymbol{D}_{v s}$ are two more parameters which we fix determined by the transport technology. We only alter the model parameters $\boldsymbol{\kappa}, \boldsymbol{\lambda}, \boldsymbol{j}$ and $\boldsymbol{\mu}$, which control the contour of the energy landscape. Values cannot be assigned to these parameters from data so most of our work involves comparing networks for different values for these inputs.

Given a definite function $\boldsymbol{H}$, we apply a Metropolis algorithm within a Monte Carlo approach to get the $\boldsymbol{N}^{2}$ variables making up our output: the site sizes $\boldsymbol{v}_{\boldsymbol{i}}$ and the link values $\boldsymbol{e}_{\boldsymbol{i j}}$. We stress that the purpose of this approach is just to provide a systematic method to calculate the minima of $\boldsymbol{H}$. This is a standard approach used in statistical methods. We start with randomly chosen values for these variables and then execute a number of 'sweeps'. In each sweep we pick each of our $\boldsymbol{N}^{2}$ variables, chosen in some random order, and try to update its value. To do this we choose a proposed new value at random, drawn with equal likelihood from the set of all allowed values. If this proposed value lowers the energy of our network the new value is kept and the network is updated. If the proposed change leaves the network at a higher energy it is only accepted with probability $\exp \left(\boldsymbol{\beta}\left(\boldsymbol{H}_{\text {old }}-\boldsymbol{H}_{\text {new }}\right)\right)$. Here $\boldsymbol{\beta}$ is a measure of the volatility. We make numerous sweeps noting how often elements are changed. If changes are happening too often, the system is too volatile; we then increase $\boldsymbol{\beta}$ and repeat. This continues until the changes are sufficiently rare, in which case we stop. At this point we now have a weighted directed network. In our visualisations we show links only if they exceed some critical value as in practice all link variables will have non-zero values but many will be very close to zero. 